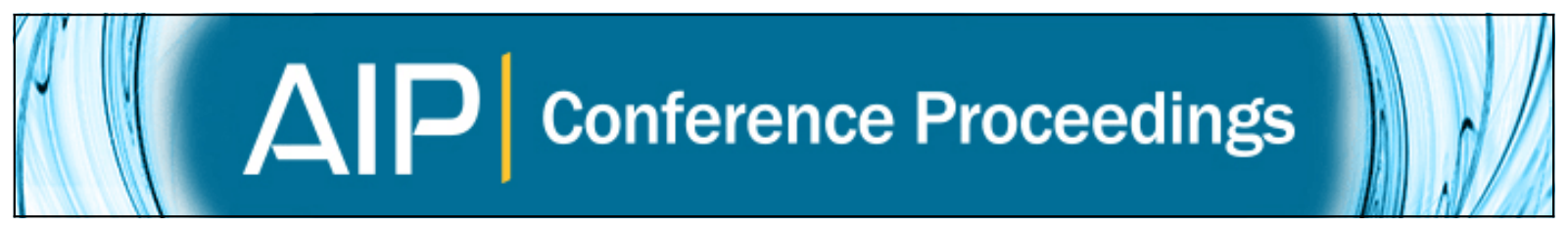

\title{
Distribution of constrained maximum coordinates
}

Martin Tláskal, Lucie Doudová, and František Buňka

Citation: AIP Conference Proceedings 1648, 720017 (2015); doi: 10.1063/1.4912954

View online: http://dx.doi.org/10.1063/1.4912954

View Table of Contents: http://scitation.aip.org/content/aip/proceeding/aipcp/1648?ver=pdfcov

Published by the AIP Publishing

Articles you may be interested in

Physically constrained maximum likelihood mode filtering

J. Acoust. Soc. Am. 127, 2385 (2010); 10.1121/1.3327799

A constrained maximum entropy method in polymer statistics

J. Chem. Phys. 119, 8162 (2003); 10.1063/1.1609197

Constrained maximum consistency multi-path mitigation

J. Acoust. Soc. Am. 114, 2448 (2003); 10.1121/1.4779409

Maximum entropy in the mean: A useful tool for constrained linear problems

AIP Conf. Proc. 659, 361 (2003); 10.1063/1.1570553

A constrained maximum entropy method for the interpretation of experimental data: Application to the derivation of single particle orientation-conformation distributions from the partially averaged nuclear spin dipolar couplings of n-alkanes dissolved in a liquid crystalline solvent

J. Chem. Phys. 105, 10595 (1996); 10.1063/1.472945 


\title{
Distribution of Constrained Maximum Coordinates
}

\author{
Martin Tláskal*, Lucie Doudová* and František Buňka ${ }^{\dagger}$ \\ ${ }^{*}$ Department of Econometrics, Faculty of Military Leadership, University of Defence in Brno, Kounicova 65, \\ 66210 Brno, Czech Republic \\ ${ }^{\dagger}$ Department of Food Technology, Faculty of Technology, Tomas Bata University in Zlín, náměstí T. G. Masaryka
}

275, 76272 Zlín, Czech Republic

\begin{abstract}
The aim of this article was to find the distribution of the random vector that determines constrained maximum coordinates of a regression function. At first the used regression function is described and the asymptotic distribution of the unconstrained maximum is derived. Then the distribution of constrained maximum coordinates is suggested for data from the food industry regarding the influence of emulsifying salts on the hardness of processed cheese.
\end{abstract}

Keywords: Delta method, emulsifying salts, processed cheese, regression analysis.

PACS: $02.50 .-\mathrm{r}$

\section{INTRODUCTION}

Processed cheese is important type of cheese which is world widely manufactured and consumed. During manufacturing, emulsifying salts should be added. Commercial emulsifying salts are mixture of 2-4 phosphates and/or citrates (especially sodium salts). Unfortunately, producers of processed cheese do not know the concrete information about composition of emulsifying salts (trade secret). The appropriate mixture of emulsifying salts (for obtaining of final products with desirable parameters) should be empirically designed. Therefore, additional costs arise. If producers have appropriate model for estimation of parameter of processed cheese using control of composition of emulsifying salts, the cost could be minimized especially at the beginning of designing of new products.

Textural parameters are one of important properties which are in focus of food industry. The hardness, cohesiveness or adhesiveness of processed cheese was modelled for example in [1], [2], [3], [4], the hardness of meat in [5]. Besides of modelling the dependence of textural parameters on important factors the aim can be to find the extreme of modelled parameter.

In this article the mixture of disodium hydrogenphosphate (DSP), tetrasodium diphosphate (TSPP) and sodium salt of polyphosphate (POLY) was used in 66 percentage proportions with a step of $10 \%(0: 0: 100,0: 90: 10,0: 80: 20$, $\cdots, 10: 0: 90, \cdots, 90: 10: 0,100: 0: 0)$ for each total concentration of emulsifying salts $(2.0 ; 2.5 ;$ and $3.0 \mathrm{~g} / 100 \mathrm{~g})$ and with or without the $\mathrm{pH}$ adjustment (to the optimal range 5.70-5.80). For details see [1]. Our aim was to find the maximum coordinates of a regression function modelling the dependence of hardness of processed cheese according to varying combination of emulsifying salts.

\section{METHODS}

Dependence of the hardness on the changing proportion of DSP (x), TSP $(y)$ and TSPP $(z)$ under the conditions $x+y+z=1$ and $x \geq 0, y \geq 0, z \geq 0$ can be generally described by general polynomial of the $m$-th order in two variables $x$ and $y$ (because $z=1-x-y$ )

$$
f(x, y)=\beta_{0}+\sum_{i=1}^{m}\left[\sum_{k=0}^{i} \beta_{i-k, k} x^{i-k} y^{k}\right],
$$

where $\beta_{0}, \beta_{1,0}, \ldots, \beta_{0, m}$ are unknown parameters. For the distribution of the random vector, that determines coordinates of the unconstrained maximum of a regression function, the Delta method was used. Then the distribution of maximum coordinates on bounded set (triangle in our case) was suggested. 


\section{On the Distribution of the Unconstrained Maximum}

For the determination of the maximum coordinates the Newton iterative method was used. The coordinates $\left(x^{*}, y^{*}\right)$ are solution of equations

$$
\sum_{i=1}^{m}\left[\sum_{k=0}^{i-1}(i-k) \beta_{i-k, k} x^{i-k-1} y^{k}\right]=0 \text { and } \sum_{i=1}^{m}\left[\sum_{k=1}^{i} k \beta_{i-k, k} x^{i-k} y^{k-1}\right]=0,
$$

which we get as partial derivatives of (1) with respect to $x$ or $y$ respectively. Let $\left(x^{*}, y^{*}\right)$ denotes the solution of (2). The random vector $\left(X^{*}, Y^{*}\right)$ depends on parameters $\beta_{0}, \beta_{1,0}, \ldots, \beta_{0, m}$.

We use the delta method (see [6]) to find the asymptotic distribution of the unconstrained maximum coordinates of a regression function on set $\mathbb{R}^{2}$. It holds $\left(X^{*}, Y^{*}\right) \stackrel{A}{\sim} N_{2}\left(\mu, \frac{1}{n} \phi^{\prime} \Sigma \phi\right)$, i.e.

$$
\left(\begin{array}{c}
x^{*}(\hat{\beta}) \\
y^{*}(\hat{\beta})
\end{array}\right) \stackrel{A}{\sim} N_{2}\left(\mu=\left(\begin{array}{c}
x^{*}(\beta) \\
y^{*}(\beta)
\end{array}\right), \frac{1}{n} \phi^{\prime} \Sigma \phi=\frac{1}{n}\left(\begin{array}{c}
\varphi^{\prime} \\
\psi^{\prime}
\end{array}\right) \Sigma(\varphi, \psi)\right),
$$

where $\varphi$ is a vector of partial derivatives of the $x$ coordinate of the maximum with respect to $\beta_{j, l}, j, l=0,1, \ldots, m$, where $1 \leq j+l \leq m$. Analogically $\psi$ is a vector of partial derivatives of $y$ coordinate of the maximum with respect to $\beta_{j, l}, j, l=0,1, \ldots, m$, where $1 \leq j+l \leq m$. Components of vectors $\varphi$ and $\psi$ are denoted $\varphi_{j, l}$, respectively $\psi_{j, l}$, $j, l=0,1, \ldots, m$, where $1 \leq j+l \leq m$. They are solution of system of linear equations which we get as partial derivatives of equations (2) with respect to $\beta_{j, l}$ :

$$
\begin{array}{ll}
j\left(x^{*}\right)^{j-1}\left(y^{*}\right)^{l}+\sum_{i=1}^{m}\left[\sum_{k=0}^{i-1}(i-k) \beta_{i-k, k}\left((i-k-1)\left(x^{*}\right)^{i-k-2}\left(y^{*}\right)^{k} \varphi_{j, l}+k\left(x^{*}\right)^{i-k-1}\left(y^{*}\right)^{k-1} \psi_{j, l}\right)\right] & =0, \\
l\left(x^{*}\right)^{j}\left(y^{*}\right)^{l-1}+\sum_{i=1}^{m}\left[\sum_{k=1}^{i} k \beta_{i-k, k}\left((i-k)\left(x^{*}\right)^{i-k-1}\left(y^{*}\right)^{k-1} \varphi_{j, l}+(k-1)\left(x^{*}\right)^{i-k}\left(y^{*}\right)^{k-2} \psi_{j, l}\right)\right] & =0,
\end{array}
$$

where $j, l=0,1, \ldots, m$ and $1 \leq j+l \leq m$.

\section{On the Distribution of the Constrained Maximum}

The coordinates of maximum represents the percentage proportions of DSP (coordinate $x$ ) and TSPP (coordinate $y$ ). The proportion of POLY is the rest to 1, i.e. the maximum will be on set given by conditions $x \geq 0, y \geq 0$ and $x+y \leq 1$. The Figure 1 illustrates this situation. The maximum of hardness can be found at an interior point of a triangle (denoted $\left.M_{0}\right)$ or can be at its vertices $\left(M_{2}^{*}, M_{4}^{*}, M_{6}^{*}\right)$ or on edges $\left(M_{1}^{*}, M_{3}^{*}, M_{5}^{*}\right)$.
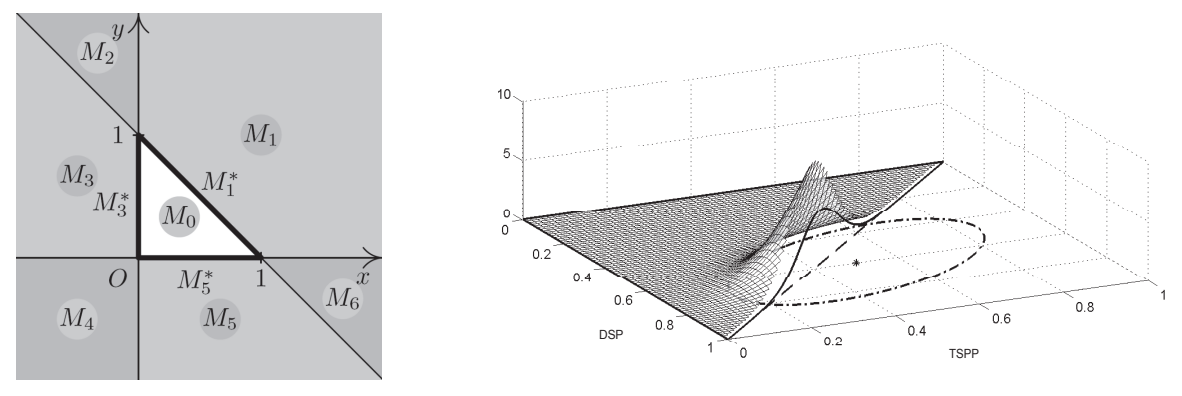

FIGURE 1. In the left figure there is shown the decomposition of set $\mathbb{R}^{2}$ to sets $M_{k}, k=0, \ldots, 6$ which are used in distribution (6). Also sets $M_{l}^{*}, l=1, \ldots, 6$ are there illustrated. The right graph shows a typical distribution of constrained maximum for the hardness. On the open triangle it has two-dimensional normal distribution and on hypotenuse it is approximated by the one-dimensional normal distribution. 
The distribution of coordinates of constrained maximum $(\tilde{X}, \tilde{Y})$ can be in general written as a mixture of twodimensional normal distributions,

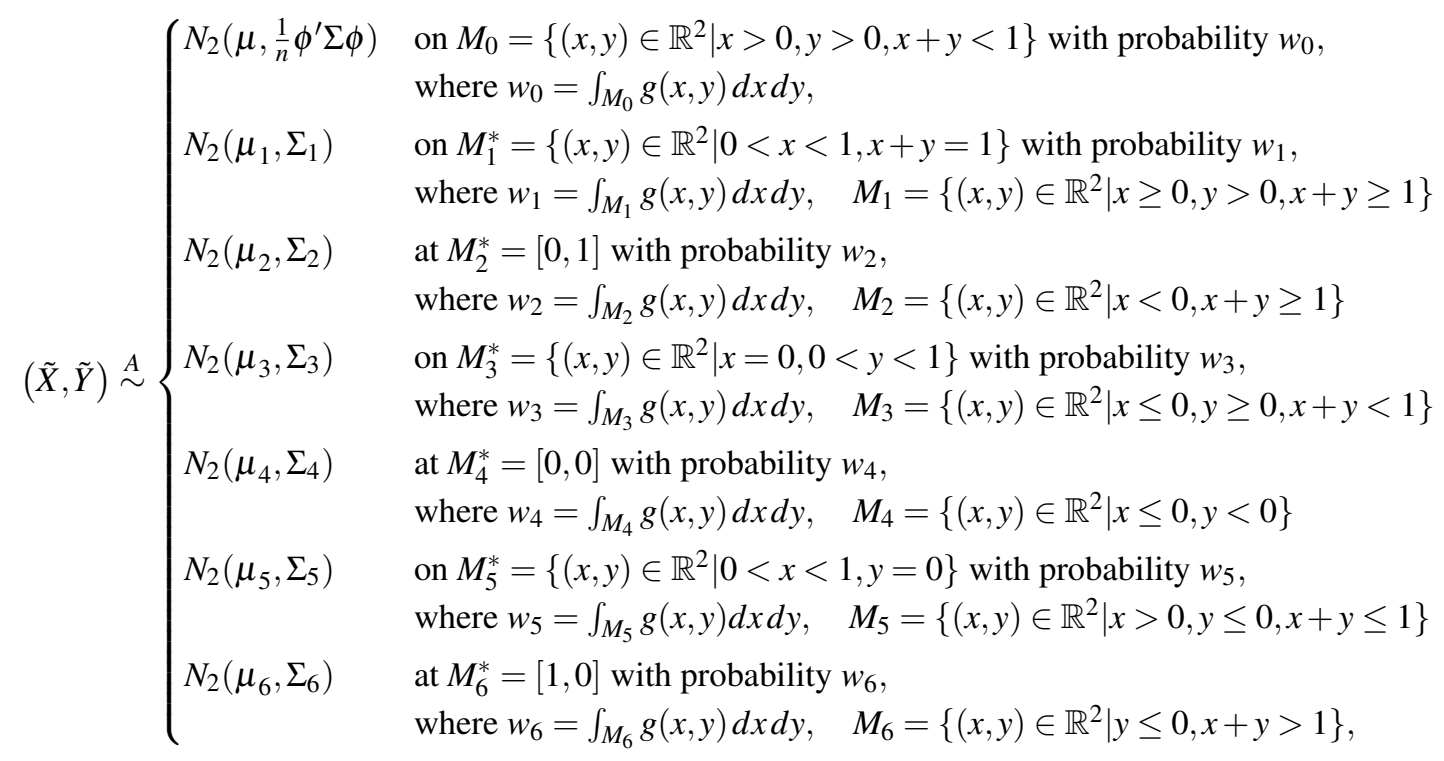

where $g(x, y)$ denotes density of two-dimensional normal distribution. If we look at individual possibilities in detail the two-dimensional normal distribution will be only on set $M_{0}$. On edges we have one-dimensional distribution and at vertices the distribution is degenerate (zero variance matrix).
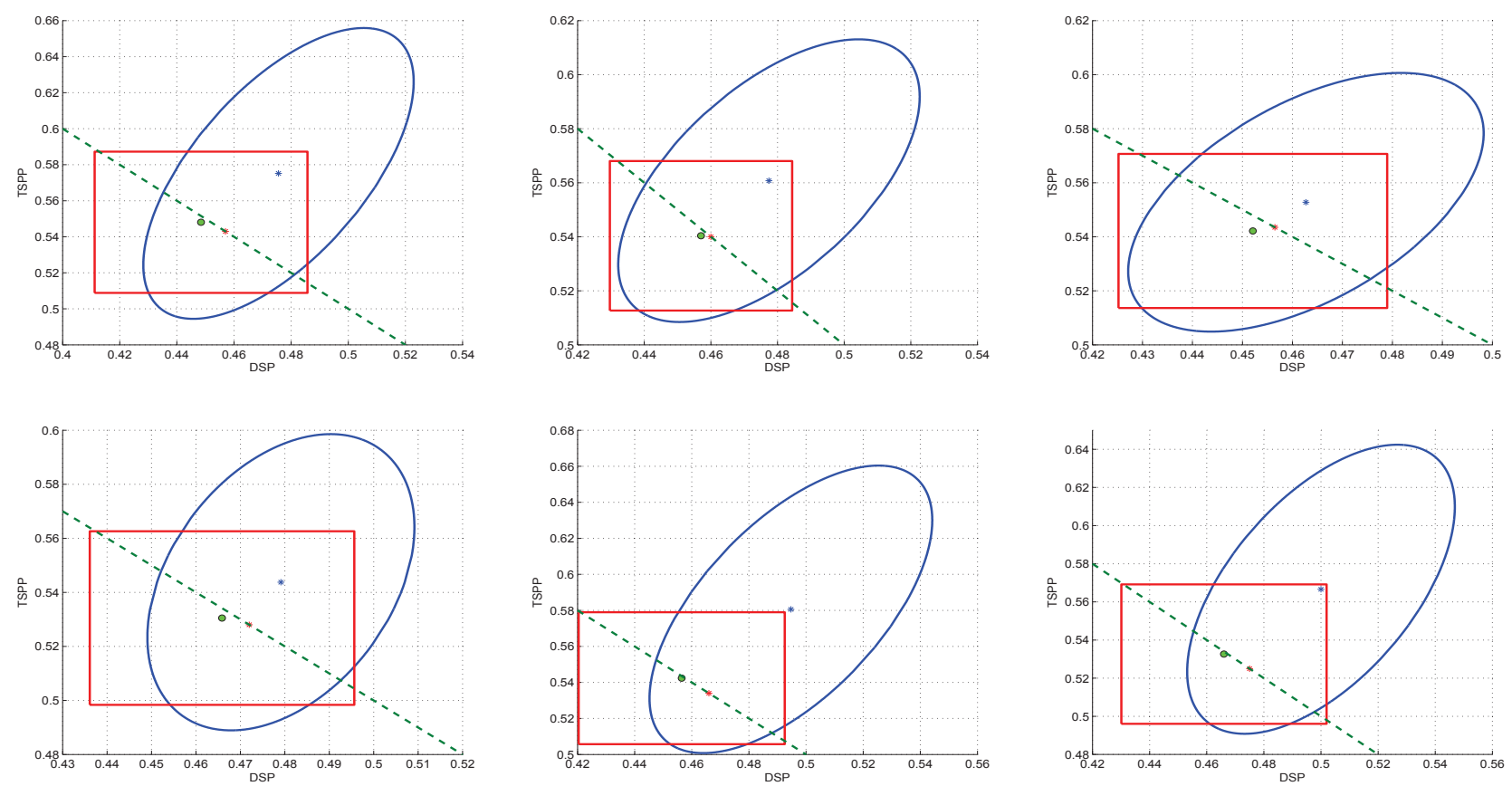

FIGURE 2. Graphic illustration of confidence sets in Table 1 . In columns concentration of phosphates (g/100 g) 2.0, 2.5, and 3.0. First row without adjustment of $\mathrm{pH}$ values, second row samples where $\mathrm{pH}$ was adjusted (to the optimal range 5.7-5.8). 


\section{RESULTS AND DISCUSSION}

All the weights in the mixture (6) were calculated numerically. In our six cases, the sum of the weights $w_{0}$ and $w_{1}$ is practically 1 , so the other weights are assessed to be zeros. Thus we consider a mixture consisting of truncated bivariate normal distribution on the set $M_{0}$ (triangle) and truncated univariate normal distribution on the set $M_{1}^{*}$ (hypotenuse of the triangle). Mean vector and variance-covariance matrix of the truncated bivariate normal distribution were computed by using the R command

mtmvnorm(mean, sigma, lower, upper)

in the R package tmvtnorm. For more details on derivation of an explicit expression see [7]. We exploited the fact, that the weights (excepting $w_{0}$ and $w_{1}$ ) are zeros and used the double rectangular truncation (over a rectangular that includes the set $M_{0}$ ). Formulas for the mean and variance of the univariate distribution can be found in [8]. Note that we used a simplification assumption again. Instead of imposing that the global maximum lies in the set $M_{1}$ we assumed that it lies in the set $M_{1}$ or $M_{2}$ or $M_{6}$. Subsequently, the normal distribution is used as a good approximation for the distribution on the set $M_{1}^{*}$. To construct the rectangular confidence area for coordinates of constrained maximum, we must determine the centre of the confidence rectangle and the proportion of its side lengths.

The centre is the mean vector of the mixture. The proportion of side lengths ( $y$ to $x$ ) is determined as $w_{0} \sigma_{Y_{t r}} / \sigma_{X_{t r}}+$ $w_{1}$, where $\sigma_{Y_{t r}}$ denotes the standard deviation of the $Y$ component of the truncated bivariate normal vector and $\sigma_{X_{t r}}$ denotes the standard deviation of the $X$ component of the truncated bivariate normal vector. To derive the confidence set an iteration process that involves numerical integration is initiated. The results are given in Table 1. The graphic illustration is shown in Figure 2. Note that the confidence set is determined as intersection of the rectangle and the triangle. Hypotenuse of the triangle is drawn by the dashed green line, the green circle denotes the centre of the rectangle (in red). The constrained maximum and the global maximum are marked by the red asterisk and by the blue asterisk, respectively. The $95 \%$ ellipse for the coordinates of global maximum is drawn in blue.

TABLE 1. The values of variables $x$ (the proportion of DSP), $y$ (the proportion of TSPP) and $z$ (the proportion of POLY) for the mean vector of the mixture (6), and $95 \%$ confidence set for the coordinates $x$ and $y$.

\begin{tabular}{|c|c|c|c|c|c|c|c|c|}
\hline \multirow{4}{*}{$\begin{array}{l}\text { Concentration } \\
\text { of phosphates } \\
(\mathrm{g} / 100 \mathrm{~g})\end{array}$} & \multirow{4}{*}{$\begin{array}{l}\text { Adjustment } \\
\text { of } \mathrm{pH} \text { values }\end{array}$} & \multirow{2}{*}{\multicolumn{3}{|c|}{ The values of variables (\%) }} & \multicolumn{4}{|c|}{ 95\% Confidence set for $(x, y)$} \\
\hline & & & & & \multicolumn{2}{|c|}{ Variable $x$} & \multicolumn{2}{|c|}{ Variable $y$} \\
\hline & & $x$ & $y$ & $z$ & Lower & Upper & Lower & Upper \\
\hline & & & & & limit & limit & limit & limit \\
\hline \multirow[t]{2}{*}{2.0} & No & 44.85 & 54.80 & 0.35 & 41.12 & 48.57 & 50.89 & 58.73 \\
\hline & Yes & 46.59 & 53.05 & 0.36 & 43.61 & 49.56 & 49.84 & 56.26 \\
\hline \multirow[t]{2}{*}{2.5} & No & 45.70 & 54.04 & 0.26 & 42.97 & 48.44 & 51.27 & 56.81 \\
\hline & Yes & 45.65 & 54.23 & 0.12 & 42.04 & 49.25 & 50.57 & 57.90 \\
\hline \multirow[t]{2}{*}{3.0} & No & 45.21 & 54.21 & 0.58 & 42.52 & 47.90 & 51.36 & 57.07 \\
\hline & Yes & 46.60 & 53.27 & 0.13 & 43.01 & 50.19 & 49.61 & 56.92 \\
\hline
\end{tabular}

\section{ACKNOWLEDGMENTS}

Supported by specific research project No. SV14-FEM-K101-01-MICH of University of Defence.

\section{REFERENCES}

1. F. Buňka, L. Doudová, E. Weiserová, M. Černíková, D. Kuchař, Š. Slavíková, G. Nagyová, P. Ponížil, T. Gruber, and J. Michálek. LWT - Food Science and Technology 58, 247-255 (2014).

2. F. Buňka, L. Doudová, E. Weiserová, D. Kuchař, P. Ponížil, D. Začalová, G. Nagyová, V. Pachlová, and J. Michálek. International Dairy Journal 29, 1-7 (2013).

3. F. Buňka, L. Doudová, E. Weiserová, D. Kuchař, J. Michálek, Š. Slavíková, and S. Kráčmar. International Journal of Food Science and Technology 47, 2063-2071 (2012).

4. E. Weiserová, L. Doudová, L. Gáliová, L. Žák, J. Michálek, R. Janiš, and F. Buňka. International Dairy Journal 21, $979-986$ (2011).

5. Š. Hanzelková, J. Simeonovová, D. Hampel, A. Dufek, and J. Šubrt, Acta Veterinaria Brno 80, 131-136 (2011).

6. A. Agresti Categorical Data Analysis. New York: Wiley, 2002.

7. B. G. Manjunath, and S. Wilhelm, (2012). Moments calculation for the doubly truncated multivariate normal density. arXiv preprint arXiv:1206.5387.

8. W. H. Greene, Econometric Analysis (5th ed.). Prentice-Hall, 2003. 